\title{
Application of the Developed Methodology for Transfer of the Temporary Dentures Parameters to a Permanent Prosthesis Supporting on Dental Implants
}

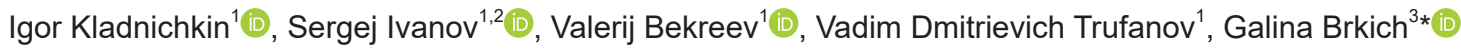 \\ ${ }^{1}$ Department of Maxillo-facial Surgery and Operative Dentistry, RUDN University, Moscow, Russian Federation, Russia; \\ ${ }^{2}$ Department of Maxillo-facial Surgery, Sechenov First Moscow State Medical University, Moscow, Russian Federation, Russia; \\ ${ }^{3}$ Institute of Translational Medicine and Biotechnology, Sechenov First Moscow State Medical University, Moscow, Russian \\ Federation, Russia
}

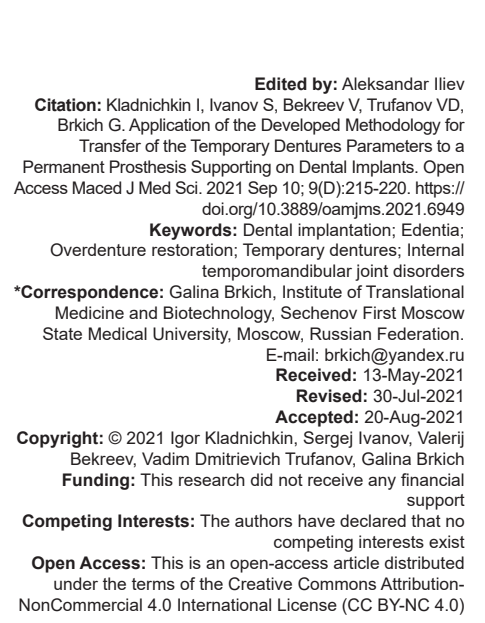

\begin{abstract}
AIM: The aim of this work is to increase the quality and accuracy of the prostheses manufacture on implants in patients with completely absent dentition, to reduce the number of doctors' visits to creation of temporary crowns, and more quickly adapt to new prostheses.

MATERIALS AND METHODS: A survey and comprehensive treatment of 55 people with complete edentulousness were carried out. Group I consisted of 30 patients who underwent treatment using the developed prototyping method, which took into account the shape of the complete removable dentures and the ratio of the jaws during the manufacture of the prosthesis on implants. The treatment of Group II, consisting of 25 patients, was carried out using a standard technique - after opening the implants, the central ratio of the jaws and the setting of the teeth were re-determined.

RESULTS: The treatment of patients with completely absent dentition using the developed prototyping methodology allowed to increase the accuracy of manufacturing temporary dentures based on dental implants, reduce the time of adaptation to the prosthesis and achieve high aesthetic results.

CONCLUSION: A prosthesis made using the proposed methodology for transferring the parameters of a temporary dentures to a permanent ones based on dental implants meets all functional and aesthetic requirements, improves the quality of prosthetics, reduces the manufacturing time of the prosthesis, the patient's adaptation time to it, and is the basis for improving the quality of patient's life.
\end{abstract}

\section{Introduction}

One of the most complex and pressing problems of modern orthopedic dentistry is a quality rehabilitation of patients with edentia. This is due to the necessity to create prostheses, which are optimal in relation of functional, esthetic, and psychological indicators of treatment results [1], [2], [3].

Methods and technologies for the correction of dentition defects are constantly being improved. Currently, one of the leading places in the system of rehabilitation of patients with edentia is dental implantation [4], [5].

Implant prostheses are made from both cermet and acrylic polymers. There are several methods of prosthetics on implants:

\footnotetext{
- $\quad$ Fixing a pre-made prosthesis on the operating table

- $\quad$ Delayed prosthetics, which are carried out in the next or remote terms after the operation of dental implantation.
}

Literature data show that in old age the methods of reconstructive plastic surgery require the most conservative approaches since in patients of this age category, somatic status is burdened with diseases that are contraindications to performing surgical operations with immediate loading of implants, narrowing the temporary prosthetics method to full integration implants [6]. However, a significant trend in modern dentistry is the desire to reduce the duration of treatment. In this regard, methods of rapid prosthetics based on dental implants are being developed and methods for their early functional load are being developed.

The results of the study suggest that prosthetics of patients with edentia on dental implants, the terms for manufacturing prostheses are reduced and their manufacturing accuracy is improved. Prosthetics for patients with complete secondary edentia who want to install implants and make prostheses based on them involves several stages. At the initial stage, a new complete removable denture (CRD) is made, on the basis of which the position and number of 
implants are planned taking into account the choice of the final orthopedic design [7], [8]. Then surgical treatment of the patient is carried out - the installation of implants [9], [10]. In case of lack of volume of the alveolar bone, preliminary or immediate bone grafting is performed.

Since the requirements for the efficiency and speed of performing dental prosthetics are increasing every year, the development of the proposed methodology is timely and relevant [11], [12].

The aim of this work is to increase the quality and accuracy of the prostheses manufacture on implants in patients with completely absent dentition, to reduce the number of doctors' visits to the creation of temporary crowns, and more quickly adapt to new prostheses.

\section{Materials and Methods}

In the clinic of the Department of Maxillo-facial Surgery and Operative Dentistry, RUDN University, and in the dental clinic IRIS, from 2017 to 2020, prosthetics of patients with edentia after reconstructive implantation operations was performed. A survey and comprehensive treatment of 55 people were carried out: 30 people in the studied Group I, who were treated according to the developed methodology for transferring the shape and the achieved ratio of the jaws according to the CRD and control Group II, consisting of 25 people who underwent prosthetics according to the standard method.

Each patient during and after the prosthetics for six months monthly underwent an ultrasound of the temporomandibular joint (TMJ) to monitor joint function. In the presence of patients' complaints of clicks, pain, discomfort in the TMJ, as well as the detection of ultrasound signs of articular displacement, they were prescribed magnetic resonance imaging (MRI) of the TMJ before and after treatment. The study was conducted on the apparatus of Sonoace R3, Samsung, Korea.

To correct occlusion of temporary and permanent prostheses, 60-micron carbon paper from Bauch, Germany was used. To adjust the thickness of the vestibular thickness of the prosthesis, $\mathrm{Re}$ Fine Yamahachi, Japan, was used. To take permanent impressions, we used individual impression trays made of Preci Tray, YETI, Germany, and Elite HD + Light and Monophase, Zhermack, Italy impression materials.

At all stages of impression taking and production of collapsible gypsum models, original impression modules for the "pick-up tray" method was used. Analogs and Scan Body and consumables for implant systems IRIS and ASTRA. Non-shrink plastic Refine Bright, Yamahachi, Japan, was used to splint the impression modules together.
For the manufacture of collapsible working plaster models, Gypsum III and IV class Fuji Rock, GC, Germany and a gingival mask Gingi Fast, Zhermack, Italy were used. For accurate scanning of gypsum models, Helling three dimensional (3D) scanning powder, Helling, Germany was used.

Milling of metal ceramics frames and beams with a MK-1 matrix was carried out from cobalt-chrome disks with a height of $15 \mathrm{~mm}$ DentaDirect, Scheftner, Germany.

The manufacture of implants bar in the creation of overdenture restoration was carried out from a powder for selective laser sintering SLS, Yeti, Germany.

The lining of metal frames was carried out by ceramic mass Ducera + Dentsply-Sirona, Germany.

For permanent closure of tap on fixed metalceramic prostheses with screw fixation, light-curing composite material Estelite Tokuyama, Dental, Japan, hydrofluoric acid 9.5\% Porcelain Etchant, Bisico, USA and All bond TE Bisico, USA was used.

Production of gypsum models from the level of multiunits or the level of implants was carried out in the dental laboratory of the IRIS clinic, Moscow. Plaster models and temporary crowns were scanned using the Shining 3D AutoScan-IT scanner to create virtual working models, as well as to create virtual models of temporary crowns. The modeling of frames, implants bar was carried out in the Exocad Matea program. Milling of frameworks for applying ceramics and production of beams with a lock MK1, Dental-Attachment Gmbh, Germany for overdenture restoration was carried out on machines of Ortos company, Russia. Selective laser sintering using an SLS apparatus, Yeti, Germany, was used to manufacture a counter beam in the manufacture of an overdenture restoration.

When lining cobalt-chrome frames in the manufacture of cermet's, layer-by-layer application of ceramic mass was used. For the final manufacture of an implants bar conditional removable prosthesis, the hot polymerization method was used with the further installation of the MK-1 lock.

\section{Results and Discussion}

The algorithm given for performing the transfer of the ratio of the jaws from the CRD to temporary prosthetics based on dental implants in patients with edentia, it was divided into the following stages.

The initial phase included the manufacture of a new CRD. Based on this prosthesis, the position and number of implants were planned taking into account the choice of the final orthopedic design. Then, surgical treatment was carried out, including implantation and, with 
a lack of alveolar bone volume, preliminary or immediate bone layer. To completion of the osseointegration of implants, they were opened and temporary prototypes based on dental implants were made by the method of prototyping a removable prosthesis.

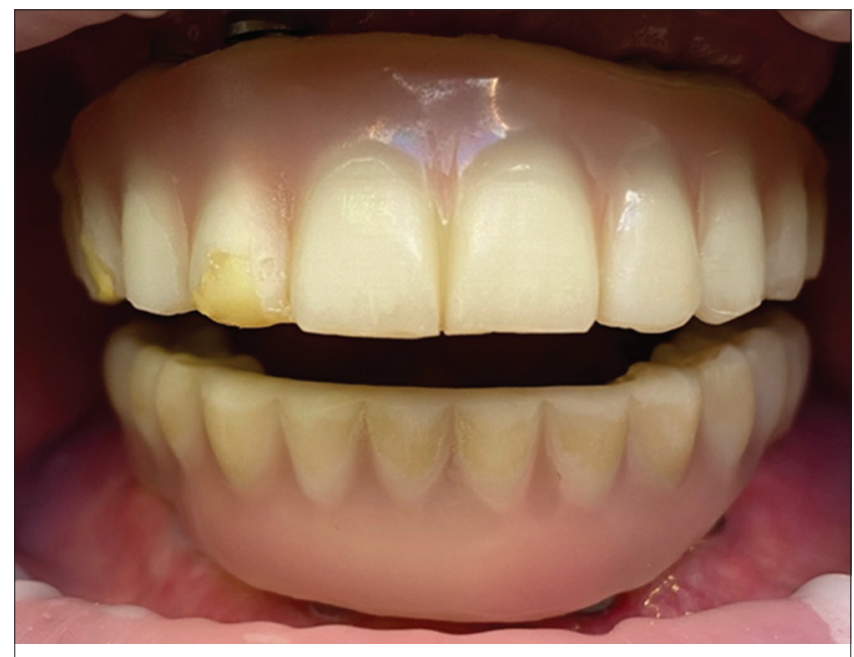

Figure 1: Front view of temporary crowns on the upper jaw

At the final stages, the patient underwent correction of temporary crowns depending on the patient's wishes related to comfort. An important part of the correction is the patient's complaints about biting the cheek, the lack of stable central occlusion. Corrections are performed by grinding supercontacts and individual points of the prosthesis's basis. Dynamic occlusion is checked. Thus, a temporary prosthesis in the form in which it becomes comfortable and aesthetic for the patient will be the prototype of a permanent prosthesis. Figures 1 and 2 show temporary crowns that will be the prototypes of permanent dentures based on dental implants.

Next, an impression was taken for the manufacture of a permanent dentures fixed on implants.

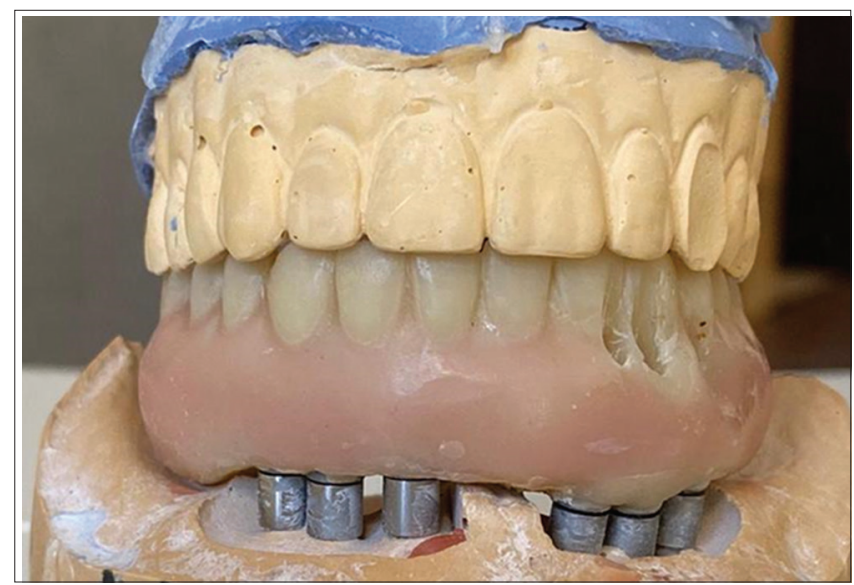

Figure 2: Front view of temporary crowns on the lower jaw

The pick-up tray was used as custom tray, relying on the mucous membrane of the denture foundation area.

After removing the temporary dentures fixed on the implants, transfers for the pick-up tray to the implants or universal abutments were installed (if overdenture restoration with fixation on the implants bar is planned). Transfers connected each other by non-shrink plastic. Next, fitting of the pick-up tray and impression taking were carried out. After that, the screws fixing the transfers were removed, the impression was removed from the oral cavity and the quality of the impression was evaluated. A new impression was also taken from the antagonizing jaw, helped to made permanent models of the jaws.

At the next patient visit, the working models were fixed in the articulator using a temporary prosthesis to which the patient was adapted. Temporary crowns were removed from the oral cavity, treated with a disinfectant solution, and mounted on a working plaster model. The models were folded together, fixed in the articulator, and reproduced the position of the jaws achieved due to temporary crowns. Temporary crowns were scanned; the file was saved in a separate patient folder. Thus, an exact virtual 3D model of temporary crowns fixed on a working gypsum model was obtained. Then, they proceeded the manufacture of permanent dentures fixed on implants using the prototyping method.

According to the proposed method, the prototype of a permanent prosthesis at this stage is temporary crowns fixed on implants. Using the form of temporary crowns, which take into account the wishes of the patient, we can begin to manufacture a permanent structure.

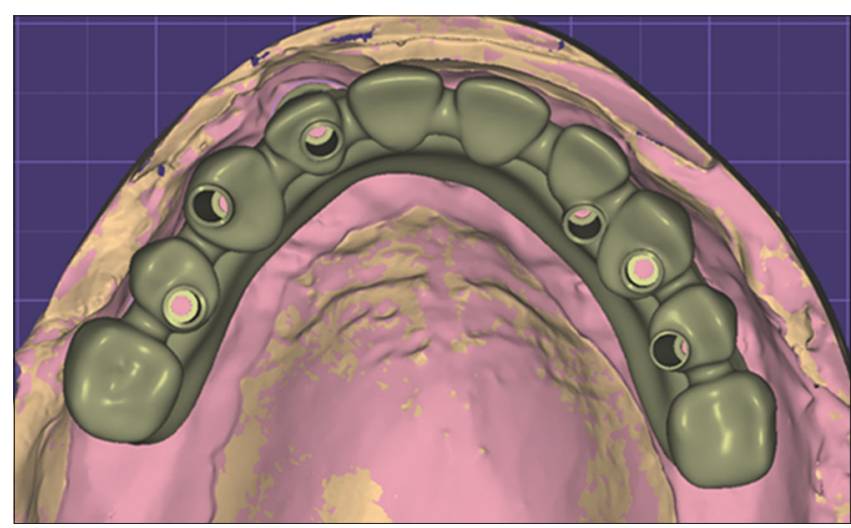

Figure 3: Three dimensional image of the frame ceramic-metal prosthesis

If the final design is a frame structure [Figure 3], then the areas of the frame that are lined with ceramics require virtual modeling with a reduction of $2 \mathrm{~mm}$ over the entire surface relative to the 3D model of temporary crowns. The reduction of $2 \mathrm{~mm}$ allows the ceramic technician to carry out the facing with ceramic mass according to the standard method, thereby minimizing the error in the performance of technical work. Figures 4 and 5 show 3D red models of temporary crowns, which are the basis for modeling the virtual framework

The frame can be made using the stl-file of zirconium dioxide by milling and subsequent sintering 


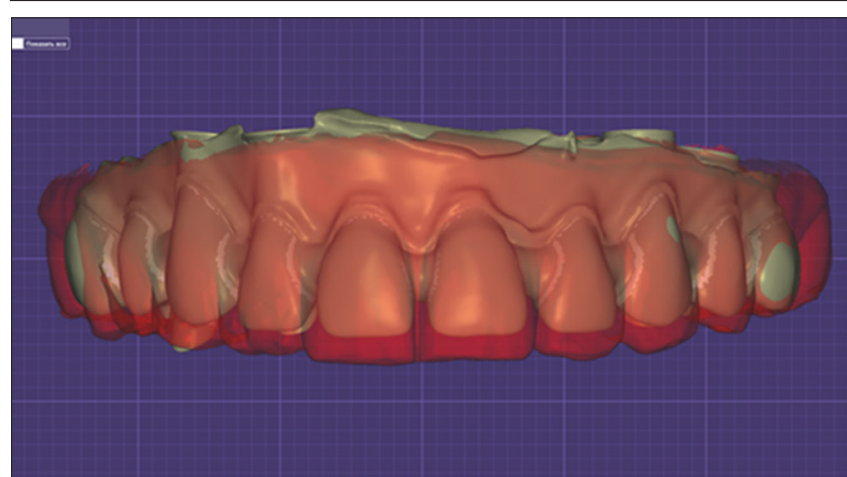

Figure 4: Three-dimensional temporary crowns with a frame applied to them, frontal view of the upper jaw

of the material, or by printing ashless photopolymer plastic and further casting the model from metal. The frame is lined with ceramic mass according to the standard method, after lining the prosthesis with its macro design is as close as possible to the temporary dentures [Figure 6].

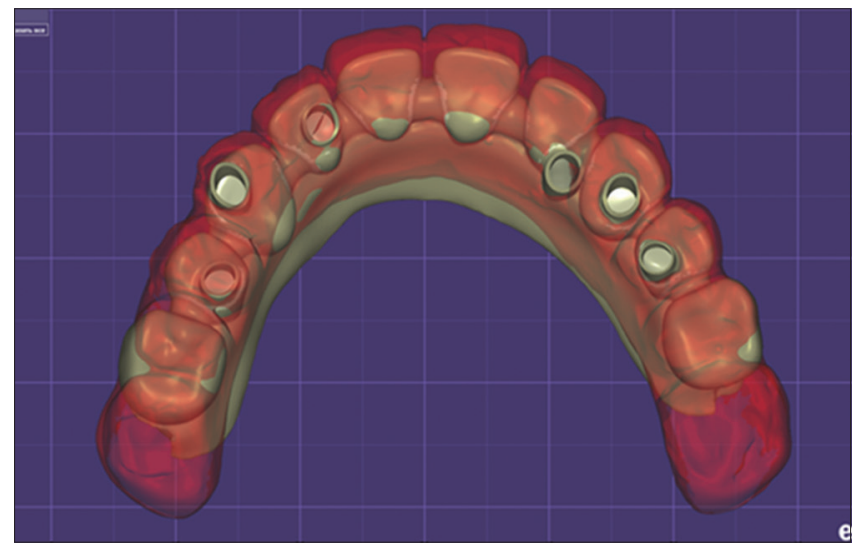

Figure 5: Three-dimensional temporary crowns with a superimposed frame

If the final design involves an implants bar conditional removable prosthesis, then the modeling of the implants bar should be also taken into account the volumes obtained from the 3D model of the patient's temporary crowns. Figure 7 shows the implants

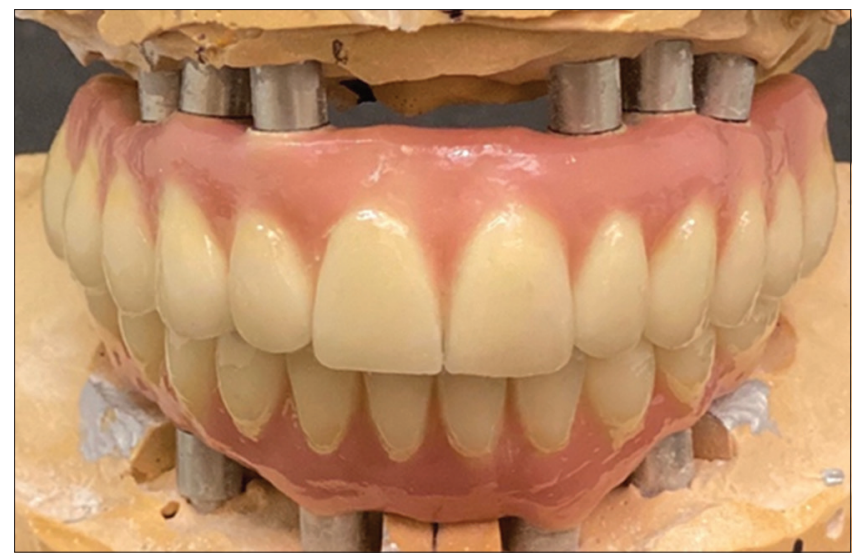

Figure 6: Finished metal-ceramic prostheses, frontal view

bar with the MK-1 lock manufactured by the milling method.

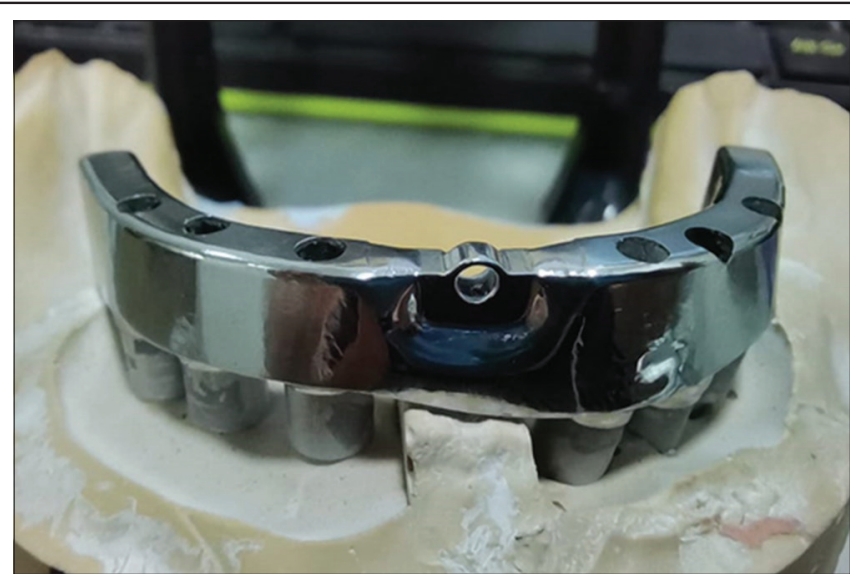

Figure 7: A beam with a MK1 lock manufactured by milling

During the simulation, the angle of inclination of the walls of the implants bar and its thickness is selected, the necessary "lock" is selected and virtually installed on the beam. After manufacturing the beam, the secondary part was made - the beam splint, which was installed on the working model and scanned. Virtual modeling was carried out in such a way that a 3D model of temporary crowns was taken into account.

If to modeling non-removable structures, a frame reduction of $2 \mathrm{~mm}$ was assumed for applying ceramic mass, and then when modeling the counter beam, the thickness under the future plastic lining of 3-3.5 mm was taken into account, respectively, the reduction of the counter beam for its modeling relative to temporary crowns. Figure 8 shows a simulated implants bar combined with a $3 \mathrm{D}$ model of temporary crowns.

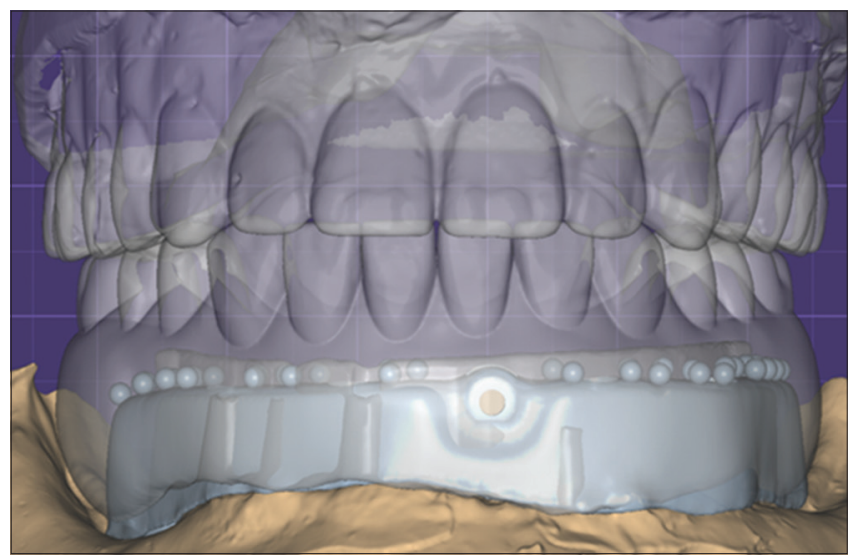

Figure 8: Three dimensional (3D) temporary crowns with $3 D$ superimposed beam splint

After fitting the implants bar on the milled beam splint, the implants bar was coated with insulating material, $3 \mathrm{~mm}$ wax lined and teeth set.

Then, using hot polymerization technology, wax was replaced with plastic. After the manufacture of the prosthesis, the operability of the lock, all functional parameters were checked. Figure 9 shows a finished semi-removable lower jaw prosthesis. 


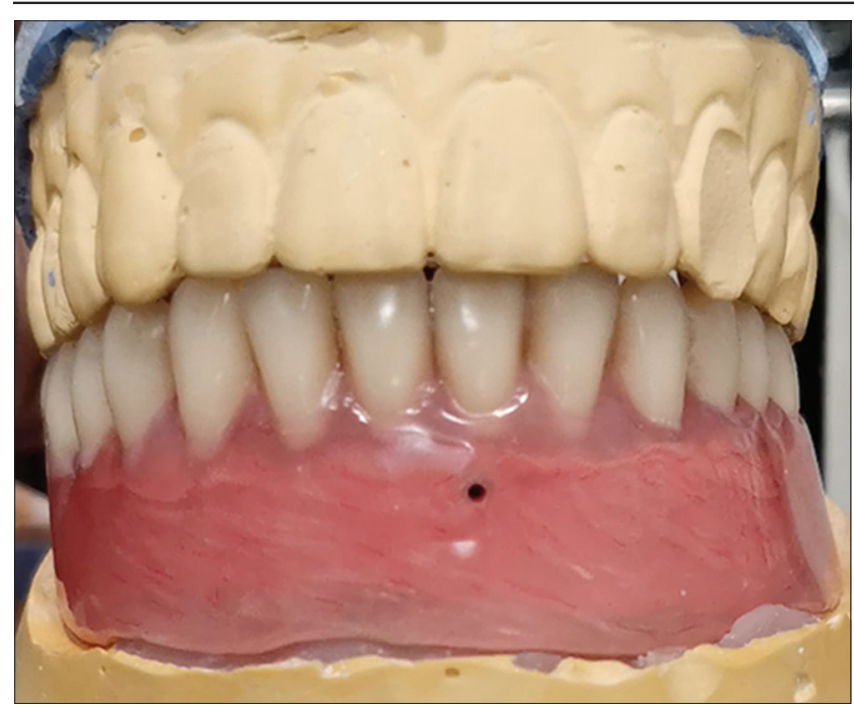

Figure 9: Finished overdenture restoration

According to MRI and ultrasound, the elimination of the TMJ disorder (TMJD) in patients of group I after prosthetics on implants according to the proposed method occurred in nine patients, the improvement of the position of the articular disk in 7 , with no effect - in 1 . The elimination of the TMJD in patients of group II after prosthetics according to the standard method in seven patients, improvement in the position of the articular disk in 5, without effect - in 2. Since the number of patients in groups I and II is different, Figure 10 shows the number of patients with TMJD disorders in percentage terms with different treatment outcomes in groups I and II.

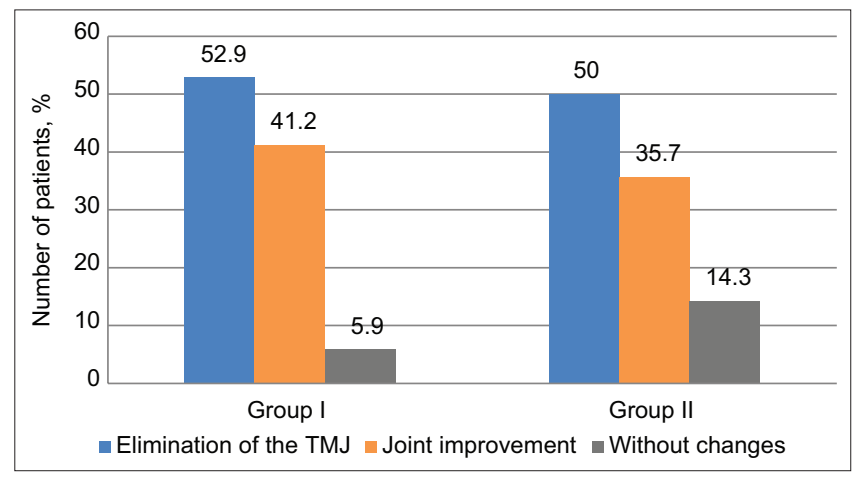

Figure 10: The number of patients with temporomandibular joint disorder as a percentage with different treatment outcomes in groups I and II

The histogram data show that the number of patients with the elimination of the TMJD and with improved joint function in the first group of patients is greater than in the second. The dynamics of normalization and improvement of the position of the articular disc in patients of groups I and II after prosthetics on implants in patients with TMJD are presented in Figure 11.

The data of the graphs show that in most patients of the I group with prosthetics on implants, an improvement in the position of the articular disc according to the developed technique occurs at the earlier date.

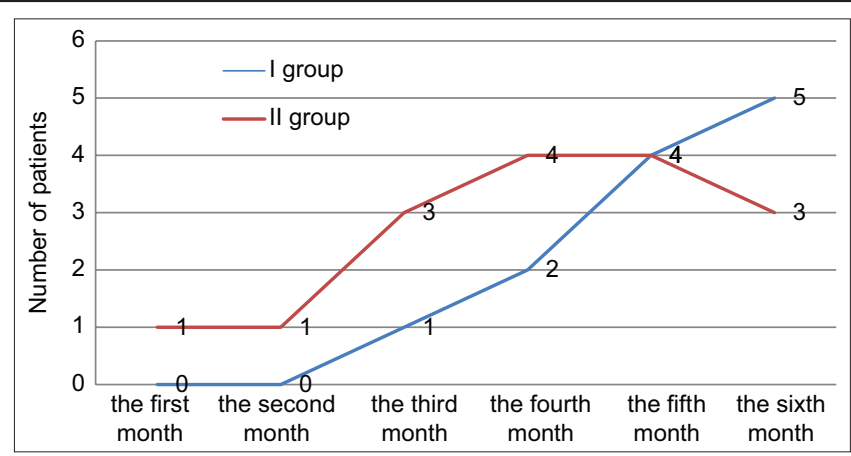

Figure 11: The dynamics of normalization and improvement of the position of the articular disc in patients of groups I and II after prosthetics on implants in patients with temporomandibular joint disorders

\section{Conclusion}

A prosthesis made using the proposed methodology for transferring the parameters of the overdenture restoration to a permanent prosthesis supporting to dental implants meets all functional and aesthetic requirements, improves the quality of prosthetics, reduces the manufacturing time of the prosthesis, the adaptation time, and is the basis for improving the quality of life of patients.

\section{References}

1. Paraskevich VL. Dental Implantology: Fundamentals of Theory and Practice. Moscow: Yunopress; 2011.

2. Dolgalev AA, Sobolev DA. Prosthetics on implants with complete loss of teeth and severe atrophy of the alveolar ridge. Dental Yug. 2008;49-50:48-51.

3. Dolgalev AA, Sobolev DA, Khubaev SZ, Tsogoev VK Prosthetics on implants in difficult anatomical conditions and in the presence of periodontitis. Periodontology. 2005;37(4):76-80.

4. Dadalyan VV. The State of Chewing Function in Patients After Prosthetics Using Implants. Moscow: Central Research Institute of Dentistry; 2006.

5. Panahov NA, Makhmudov TG. The level of stability of dental implants at different periods of functioning. Prob Dent. 2018;14(1):89-93.

6. Buchnev DY. Optimization of Surgical Tactics Fordental Implantation. Moscow: Moscow State Medical and Dental University; 2006.

7. Ivanov SY, Solodky VG, Muraev AA, Starostin PV. Russian system of dental implants LIKO-M: Experience of five years of clinical use. Dentistry. 2013;6:53-55.

8. Kladnichkin ID. Denture treatment with support for implants. In: Scientific Research of the SCO Countries: Synergy and Integration: Materials of the International Conference, Part 1. Beijing: PRC, Scientific Publishing House Infinity; 2020. p. 108-15.

9. Shashmurina VR. Mechanisms for Adapting Patients to Dentures Based on Implants in the Complete Absence of Teeth in the Lower Jaw. Moscow: Institute for Advanced Studies of the Federal Medical and Biological Agency of 
Russia; 2008. p. 90-5.

10. Dobrovolskaya OV, Dobrovolsky AV, Rubanenko VV Performance comparison rehabilitation of patients with full adentia of the lower jaw using various design options based on implants. Ukr Dent Almanac. 2011;3:17-8.

11. Kakachi K, Neugebauer J. Handbook of Dental Implantology.
Moscow: MEDpress-Inform; 2009. p. 207.

12. Bykovskaya TV, Ivanov SY, Korotkova NL, Muraev AA, Bekreev VV, Safyanova EV, et al. Assessment of the temporomandibular joint during orthognathic surgery for congenital malformations of the jaw without using a surgical template. Head Neck. 2018;1:23-8. 